\title{
DISCONTINUITY OF THE PRODUCT IN MULTIPLIER ALGEBRAS
}

\author{
M. OUDADESS
}

\begin{abstract}
Entire functions operate in complete locally $A$-convex algebras but not continuously. Actually squaring is not always continuous. The counterexample, we give, is a multiplier algebra.
\end{abstract}

\section{Introduction}

W. Żelazko constructs ([13]) an example of a non $m$-convex algebra on which all entire functions operate. Doing so he solves a problem stated in [12]. The example turns out to be a uniformly $A$-convex algebra. The problem in question had also been solved in [10].

We obtain that entire functions do not operate continuously. Actually we show by a counter-example, which is a multiplier algebra, that the product is not (globally) continuous in general. On the other hand we obtain here that it is always sequentially continuous in any unital and conplete locally $A$-convex algebra. In the Uniformly $A$-convex case it is hypocontinuous.

\section{Sequential continuity and hypocontinuity of the product}

Let $E$ be a locally convex algebra with a topology defined by the family of semi-norms $\left(p_{\lambda}\right)_{\lambda \in \Lambda}$. Then $E$ is said to be locally $A$-convex ([4]) if for every $x \in E$ and every $\lambda \in \Lambda$ there exist $M(\lambda, x)>0, N(\lambda, x)>0$ such that

$$
\begin{aligned}
& p_{\lambda}(x y) \leq M(\lambda, x) p_{\lambda}(y) \quad(y \in E) . \\
& p_{\lambda}(y x) \leq N(\lambda, x) p_{\lambda}(y) \quad(y \in E) .
\end{aligned}
$$

A locally $A$-convex aigebra is said to be Uniformly $A$-convex algebra if $M(\lambda, x)$ and $N(\lambda, x)$ can be chosen independently of $\lambda([5])$.

Observe that the so-called locally $m$-convex algebras ([9]) are particular cases of the above definition. Some examples of all these classes of algebras and relationships among them can be seen in ([4]) and ([5]). 
Let $E$ be a commutative Banach algebra without order i.e if $x y=0(y \in E)$ then $x=0$. The multiplier algebra $M(E)$ of $E$ is the space of linear operators $T$ verifying $T(x . y)=x T(y)(x, y \in E)$. Endowed with the strong topology given by the family of semi-norms $\left(P_{x}\right)_{x \in E}$, where $p_{x}(T)=\|T x\|(x \in E), M(E)$ is a unital, complete, uniformly locally $A$-convex algebra which is not always $m$ convex since it is a generalization (see $\left[1\right.$, p. 139]) of the algebra $C_{b}(R)$, of $[4]$, that is not $m$-convex.

We can endow any unital locally $A$-convex algebra with a locally $m$-convex topology $M(\tau)$ fner than $\tau$ by putting $q_{\lambda}(x)=\sup \left\{p_{\lambda}(x . y): p_{\lambda}(y) \leq 1\right\}$. This topology is complete when $\tau$ is. Moreover $\tau$ and $M(\tau)$ have the same bounded sets $([2])$; indeed it suffices to show that bounded sets for $\tau$ are bounded for $M(\tau)$, but this follows from the fact that any barrel in a complete locally convex space is bornivorous.

Proposition 2.1. Entire functions operate on any unital and compete locally A-conver algebra.

Proof: $(E, M(\tau))$ is a unital complete locally $m$-convex algebra and entire functions operate on such algebras $([9])$; the result follows since $\tau$ is coarser than $M(\tau)$.

Proposition 2.2. In any unital and complete locally A-convex algebra $(E, \tau)$, the product is always sequentially continuous.

Proof: Let $\left(x_{n}\right)_{n}$ and $\left(y_{n}\right)_{n}$ be two sequences converging to zero in $(E, \tau)$. Since $\tau$ and $M(\tau)$ have the same bounded sets, $\left(q_{\lambda}\left(x_{n}\right)\right)_{n}$ is bounded for every $\lambda$. Then the conclusion follows from the relation $p_{\lambda}(x \cdot y) \leq q_{\lambda}(x), p_{\lambda}(y)$, for every $x$ and $y$.

We can endow any unital and complete uniformiy $A$-convex algebra $(E, \tau)$ with a Banach algebra norm $\|$. $\|$ finer than $\tau$, by putting $\|x\|=\sup \left\{q_{\lambda}(x)\right.$ : $\lambda \in A\} ;$ it is Cochran's norm $([5])$. And, as before, we can show that $\tau$ and $\|\cdot\|$ have the same bounded sets. This has, as a consequence, the proposition 2 of [13].

Now recall that, in a locally convex algebra $(E, T)$. The multiplication is said to be left (right) hypocontinuous if for each neighborhood $U$ of $O$ and any bounded set $B$ there exists a neighborhood $V$ of o such that $B . V \subset U(V . B \subset$ $U)$. The multiplication, in $E$, is called hypocontinuous if it is left as well as right hypocontinuous.

Proposition 2.3. In a unital and complete uniformly A-convex algebra $(E, \tau)$ the product is always hypocontinuous.

Proof: By ([6, proposition 9, p. 155]) it is sufficient to prove that if we consider the map $u: x \rightarrow L_{x}$, where $L_{x}(y)=x y(x, y \in E)$ then, for every 
bounded set $B$ in $E$, the set $u(B)$ is equicontinuous. But this follows from the relation $p_{\lambda}(x y) \leq\|x\| \cdot p_{\lambda}(y)(x, y \in E, \lambda \in \Lambda)$ and the fact that $\tau$ and $\|\cdot\|$ have the same bounded sets.

\section{Discontinuity of the product}

Our counter-example is the multiplier algebra of an $H^{*}$-algebra. It goes along the lines of ([7, problem 111]).

An $H^{*}$-algebra is a Banach algebra $E$, with involution $*$, which is a Hilbert space under a scalar product $\langle.$,$\rangle such that.$

a) $\|x\|^{2}=\langle x, x\rangle$, for every $x$ in $E$

b) $\left\|x^{*}\right\|=\|x\|$, for every $x$ in $E$

c) $x^{*} \cdot x \neq 0$, for every $x$ in $E \backslash\{0\}$.

d) $\langle x . y, z\rangle=\left\langle y, x^{*} . z\right\rangle=\left\langle x, z . y^{*}\right\rangle$, for every $x, y, z$ in $E$.

In such commutative algebras there always exists a complete orthogonal system of idempotent and self-adjoint elements (we can even suppose more but this is sufficient for our needs; $c f[3]$ ).

Counter-example 5.1. Let $(E,\|\cdot\|)$ be a commutative infinite dimensional $H^{*}$-algebra. Without loss of generality suppose $E$ separable. Let then $\left(e_{1}, e_{2}, \ldots, e_{k} \ldots\right)$ be a complete orthogonal system of self-adjoint and indempotent elements. The set $\left\{\sqrt{k} \cdot e_{k}: k=1,2, \ldots\right\}$ is unbounded since $\left\|e_{k}\right\| \geq$ $(k=1,2, \ldots)$ and it contains the mull vector in its weak closure (cf., $[7$, problem 28]). Hence there exists a net $\left(k_{i}\right)_{i}$ of positive integers such that $\sqrt{k_{i}}, e_{k_{i}}$ converges weakly to zero (it cannot be a sequence).

For each $k$, consider the multiplier $A_{k}$ defined by $A_{k}(x)=k^{\prime} \cdot e_{k}, x$ where $k^{t}$ is the fourth root of $k$. We have $\left\|A_{k_{i}}(x)\right\|^{2}=\left\langle\sqrt{k_{i}} . e_{k_{i}}, x . x^{*}\right\rangle$, hence $\left\|A_{k_{i}}(x)\right\| \rightarrow$ 0 . But $A_{k_{i}}^{4}(x)=k_{i} \cdot e_{k_{i}} \cdot x$, and. if therefore we take in particular $x=\left(1, \ldots, n^{-1}\right.$, $\ldots)$, then $A_{k_{i}}^{4}(x)=e_{k_{i}}$. So $\left\|A_{k_{i}}^{4}(x)\right\| \geq 1$. Whence squaring is not continuous.

Remarks.

1. In many interesting situations the product is continuous. This is the case for the example $C_{b}(R)$ of [4]. It is also so for any multiplier algebra $M(E)$ where $E$ admits factorization, i.e for every $z \in E$, there exist $x$ and $y$ in $E$ such that $z=x . y$.

2. In connection with the previous remark, we can notice that there cannot exist a Banach algebra $E$ admitting a bounded approximate identity and such that the set $N=\left\{x: x^{2}=0\right\}$ is $\beta$-dense in $E$. Indeed the product, and in particular squaring, is continuous, hence $\left\{T e M(E): T^{2}=0\right\}$ in $\beta$-closed. It is also $\beta$-dense since it contains $N$ and $E$ is $\beta$-dense in $M(E)$ for it admits a bounded approximate identity. Therefore any element of $M(E)$ should be nilpotent. But this contradicts the fact that $M(E)$ is always unital.

3. Incidentally we get that an absolutely convergent series in a complete locally convex space does not necessarily define a continuous mapping. Indeed if $(E, \tau)$ is a locally uniformly $A$-convex algebra whose topology $\tau$ is given by a 
family of seminorms $\left(P_{\lambda}\right)_{\lambda}$, there exists a Banach algebra norm $\|\cdot\|$ and $\alpha>0$ such that $P_{\lambda}(x) \leq \alpha$. $\|x\|$, for every $\lambda$ and every $x$. Now if $f(z)=\sum a_{n} \cdot z^{n}$ is an entire function, then, for every $\lambda, P_{\lambda}\left(\sum a_{n} \cdot x^{n}\right) \leq \alpha \cdot \sum\left|a_{n}\right| \cdot|| x \|^{n}$. Hence the series is absolutely convergent; but the previous counter-example shows that the map $x \rightarrow f(x)$ is not always continuous.

Acknowledgments. I'm indebted to the referee for having detected an error in the first version and having suggested many improvements for the writing of the second one.

\section{References}

[1] M. AKKAR, "Etude Spectrale et structures d'algèbres topologiques et bornologiques complétes," Thèse d'état, Univ. de Bordeaux I, 1976.

[2] M. Akkar, L. OUbBi and M. Oudadess, ALgèbres $A$-convexes et problème de Michaël, Proc. A.M.S. (à paraître).

[3] F.F. BONSAll AND J. DunCAN, "Complete normed algebras," Ergebnisse der Mathematik, Band 80, Springer-Verlag, 1973.

[4] A.C. Cochran. R. Keown and C.R. Williams, On a class of topological algebras, Pacific J. Math. 34 (1970), 17-25.

[5] A.C. Cochran, Representation of A-convex algebras, Proc. Amer. Math. Soc. 41 (1973), 473-479.

[6] A. Grothendieck, Espaces vectoriels topologiques, Pub. Soc. Math., S. Paulo (1964).

[7] P.R. Halmos, "A Hilbert space problem book," Second Ed., Springer-Verlag, 1974.

[8] R. LARSEN, "The multiplier problem," Lect. Notes Math. 105, SpringerVerlag, 1969.

[9] E.A. Mıснӓ̈L, Locally multiplicatively convex topological algebras, Memoirs Amer. Math. Soc. 11, Providence (1952).

$\{10\} \mathrm{M}$. OUdADEss, Théorèmes de structures et propriétés fondamentales des algèbres uniformément $A$-convexes, C.R. Acad. Sci. Paris 296, Série I (1983), 851-853.

[11] J.K. WANG, Multipliers of commutative Banach algebras, Pacific $J$. Math. (1961), 1131-1149. 
[12] W. $\dot{Z}$ ELAZKo, Selected topics in topological algebras, Lect. Notes séries 31 (1971), Matematisk Institut, Aarhus Universitet.

[13] W. ŻELAZKo, A non $m$-convex algebra on which operate all entire functions, Ann. Polinici Math. 46 (1985), 389-394.

Ecole Normale Supérieure Takaddoum

B.P. 5118 Rabat

MAROC

Rebut el 26 d'Abril de 1990 